\title{
A singular position-dependent mass particle in an infinite potential well
}

\author{
Omar Mustafa $^{1}$ and S.Habib Mazharimousavi ${ }^{2}$ \\ Department of Physics, Eastern Mediterranean University, \\ G Magusa, North Cyprus, Mersin 10,Turkey \\ ${ }^{1}$ E-mail: omar.mustafa@emu.edu.tr \\ Tel: +903926301314 \\ Fax: +903923651604 \\ ${ }^{2}$ E-mail: habib.mazhari@emu.edu.tr
}

October 30, 2018

\begin{abstract}
An unusual singular position-dependent-mass particle in an infinite potential well is considered. The corresponding Hamiltonian is mapped through a point-canonical-transformation and an explicit correspondence between the target Hamiltonian and a Pöschl-Teller type reference Hamiltonian is obtained. New ordering ambiguity parametric setting are suggested.
\end{abstract}

PACS codes: 03.65.Ge, 03.65.Ca

Keywords: Position-dependent-mass, point-canonical-transformation, Pöschl-Teller potential, ordering ambiguity. 


\section{Introduction}

In addition of being a descriptive model for some physical phenomena [1-36] (including but not limited to, energy density many-body problem, electronic properties of semiconductors), the position-dependent-mass (PDM) von Roos Hamiltonian [33] (in $\hbar=2 m_{\circ}=1$ units)

$$
H=-\frac{1}{2}\left[m(x)^{\alpha} \partial_{x} m(x)^{\beta} \partial_{x} m(x)^{\gamma}+m(x)^{\gamma} \partial_{x} m(x)^{\beta} \partial_{x} m(x)^{\alpha}\right]+V(x)
$$

is shown to be a mathematically useful model as it enriches the class of exactlysolvable quantum mechanical problems. Whilst the parameters $\alpha, \beta$, and $\gamma$ are subjected to the so-called von Roos constraint

$$
\alpha+\beta+\gamma=-1 ; \alpha, \beta, \gamma \in \mathbb{R}
$$

the only feasibly admissible case that ensures the continuity conditions at the heterojunction boundaries between two crystals is for $\alpha=\gamma$. This, in effect, reduces the domain of the acceptable values of the so-called "ambiguity parameters" $\alpha, \beta$, and $\gamma$ and suggests that the PDM-Hamiltonian (1) be rewritten as

$$
H=-m(x)^{\alpha} \partial_{x} m(x)^{\beta} \partial_{x} m(x)^{\alpha}+V(x) ; 2 \alpha+\beta=-1 .
$$

Recently, moreover, Dutra and Almeida [14] have carried out a reliability test on the orderings available in the literature. They have used an exactly solvable Morse model and concluded that the orderings of Gora and Williams [15] $(a=\beta=\gamma=0, \alpha=-1)$, and Ben Danial and Duke [16] $(a=\alpha=\gamma=0$, $\beta=-1$ ) should be discarded for they result in complex energies. Nevertheless, they have classified the ordering of Zhu and Kroemer [17] $(a=0, \alpha=\gamma=-1 / 2$, $\beta=0)$, and that of Li and Kuhn [18] $(a=\alpha=0, \beta=\gamma=-1 / 2)$ as good 
orderings. Therefore, the continuity conditions at the heterojunction boundaries and Dutra's and Almeida's [14] reliability test would ultimately single out Zhu and Kroemer ( $a=0, \alpha=\gamma=-1 / 2, \beta=0)$ as a "reliable good ordering" . Fitting into this category/classification, Mustafa and Mazharimousavi [19] have used a PDM-pseudo-momentum operator and suggested a new "reliable good ordering" ( $\beta=-1 / 2, \alpha=\gamma=-1 / 4)$. Yet, in their study of classical and quantum PDM harmonic oscillator, Cruz et al [20] have considered different ambiguity parameters settings and argued that only one of these orderings, $\beta=-1 / 2, \alpha=\gamma=-1 / 4$ (i.e., that of Mustafa and Mazharimousavi [19]), gives rise to a potential term that is the same as the classical PDM oscillator. For more details on the ambiguity associated with the uniqueness of the kinetic energy operator, the reader my refer to $[8,14,19,20$ and references cited therein].

In this letter, only for mathematical and/or quantum mechanical curiosity on the associated exact-solvability, we consider the PDM von Roos Hamiltonian (1) for a quantum particle moving within the domain mandated by its own PDM-function $m(x)$ accompanied by an infinite potential well $V(x)$. In section 2, we consider an unusual PDM-function of the form [20]

$$
m(x)=\frac{m_{\circ}}{\left[1-\left(\frac{x}{a}\right)^{2}\right]^{2}} ; V(x)=\left\{\begin{array}{l}
0 \text { for }|x|<a \\
\infty \text { for }|x| \geq a
\end{array} .\right.
$$

Under such settings, it is obvious that the classical motion of such a particle is confined to the domain $\mathcal{D}(x)=(-a, a)$. and the PDM-Hamiltonian in (1) would result a PDM-Schrödinger equation of the form

$$
\left[-\partial_{x} \frac{1}{m(x)} \partial_{x}+\tilde{V}(x)\right] \psi(x)=E \psi(x) ; \quad|x|<a,
$$


with

$$
\tilde{V}(x)=\frac{1}{2}(1+\beta) \frac{m^{\prime \prime}(x)}{m(x)^{2}}-[\alpha(\alpha+\beta+1)+\beta+1] \frac{m^{\prime}(x)^{2}}{m(x)^{3}}+V(x) .
$$

Where primes denote derivatives with respect to $x$ and the PDM-Schrödinger equation in (5) is known as the target equation. Obviously, as $\alpha, \beta$, and $\gamma$ change within constraint (2), a profile change of $\tilde{V}(x)$ in (6) is unavoidable (hence, an ordering ambiguity conflict erupts in the process). Consequences of a pointcanonical-transformation (PCT) mapping (often mediates a transition between two different effective potentials) on such a PDM particle are also discussed in section 2. Therein, we shall witness that such a mass setting results in an effective reflectionless modified Pöschl-Teller type potential well (cf. e.g., [20, 37]). We conclude in section 3 .

\section{Consequences of PCT-mapping}

Following the well known PCT recipe (cf., e.g., [19,24;27]) would, with a substitution of the form $\psi(x)=m(x)^{1 / 4} \phi(q(x))$ in $(5)$, result in $q^{\prime}(x)=\sqrt{m(x)}$ and suggests the following point canonical transformation

$$
q(x)=\int^{x} \sqrt{m(t)} d t=a \tanh ^{-1}\left(\frac{x}{a}\right) ; \quad|x|<a,
$$

with $m(x)$ given in (4). Consequently, the PDM-Schrödinger equation (5) is mapped into

$$
\left(-\frac{d^{2}}{d q^{2}}+V_{e f f}(q)\right) \phi(q)=E \phi(q) ; q \in(-\infty, \infty)
$$


where

$$
V_{e f f}(q)=g_{1} \frac{m^{\prime \prime}(x)}{m(x)^{2}}-g_{2} \frac{m^{\prime}(x)^{2}}{m(x)^{3}},
$$

with

$$
g_{1}=\frac{1}{4}(1+2 \beta), \quad g_{2}=\alpha(\alpha+\beta+1)+\beta+\frac{9}{16} .
$$

One may then use (7) and substitute

$$
\frac{x}{a}=\tanh \left(\frac{q}{a}\right) ; \quad|x|<a,
$$

in (9) to imply

$$
V_{e f f}(q)=-\frac{\lambda(\lambda-1)}{a^{2} \cosh ^{2}(q / a)}+\frac{8}{a^{2}}\left(3 g_{1}-2 g_{2}\right) ; \lambda(\lambda-1)=4\left(5 g_{1}-4 g_{2}\right) .
$$

Which, in turn, would lead to an over simplified reference Schrödinger equation of the form

$$
\frac{d^{2} \phi(q)}{d q^{2}}+\left(k^{2}+\mu^{2} \frac{\lambda(\lambda-1)}{\cosh ^{2}(\mu q)}\right) \phi(q)=0,
$$

where

$$
k^{2}=E-8 \mu^{2}\left(3 g_{1}-2 g_{2}\right)=\mathcal{E} ; \mu=1 / a
$$

Remarkably, the "shifted-by-a-constant" potential well in (12) is known to be reflectionless/transparent (at any energy) with a reflection coefficient equals zero if $\lambda>1$ is a positive integer (cf., e.g., Lekner [21] and/or Diaz et al. [25] for a comprehensive and detailed study on this potential). Therefore, a quick recollection to the essentials of the Modified Pöschl-Teller potential by Diaz et al. [25] would (with a bound state solution when $\mathcal{E}<0$ ) provide a clear 
correspondence to the following recycled solution

$$
\begin{aligned}
\phi(q)= & (\cosh \mu q)^{\lambda}\left[A_{2} F_{1}\left(a, b ; \frac{1}{2} ;-\sinh ^{2} \mu q\right)+\right. \\
& \left.B(\sinh \mu q)_{2} F_{1}\left(a+\frac{1}{2}, b+\frac{1}{2} ; \frac{3}{2} ;-\sinh ^{2} \mu q\right)\right]
\end{aligned}
$$

where

$$
a=\frac{1}{2}\left(\lambda-\frac{\sqrt{|\mathcal{E}|}}{\mu}\right) ; \quad b=\frac{1}{2}\left(\lambda+\frac{\sqrt{|\mathcal{E}|}}{\mu}\right)
$$

and

$$
\mathcal{E}_{n}=-\mu^{2}(\lambda-1-n)^{2} ; \quad n \in \mathbb{N}, 0 \leq n<\lambda-1
$$

Hereby, as long as the reference Schrödinger equation (13) is in point, the corresponding solutions hold true. However, a mapping to the original target problem defined through equations (4)-(6) would suggest that

$$
\begin{aligned}
\psi_{n}(x)= & \left(\frac{1}{1-\left(\frac{x}{a}\right)^{2}}\right)^{\frac{\lambda+1}{2}}\left[A_{2} F_{1}\left(a, b ; \frac{1}{2} ; \frac{\left(\frac{x}{a}\right)^{2}}{\left(\frac{x}{a}\right)^{2}-1}\right)\right. \\
& \left.+B\left(\sqrt{\frac{\left(\frac{x}{a}\right)^{2}}{1-\left(\frac{x}{a}\right)^{2}}}\right){ }_{2} F_{1}\left(a+\frac{1}{2}, b+\frac{1}{2} ; \frac{3}{2} ; \frac{\left(\frac{x}{a}\right)^{2}}{\left(\frac{x}{a}\right)^{2}-1}\right)\right],
\end{aligned}
$$

and

$$
E_{n}=\mu^{2}\left(8\left(3 g_{1}-2 g_{2}\right)-(\lambda-1-n)^{2}\right), \quad n \in \mathbb{N}, 0 \leq n<\lambda-1,
$$

with

$$
a=\frac{1}{2}(1+n) ; \quad b=\lambda-\frac{1}{2}(1+n) .
$$


The ground state solution of which would (with $n=0$ ) read

$$
\begin{aligned}
\psi_{0}(x) & =A_{0}\left(\frac{1}{1-\left(\frac{x}{a}\right)^{2}}\right)^{(\lambda+1) / 2} F\left(\frac{1}{2}, \lambda-\frac{1}{2} ; \frac{1}{2} ; \frac{\left(\frac{x}{a}\right)^{2}}{\left(\frac{x}{a}\right)^{2}-1}\right) \\
& =A_{0}\left(\frac{1}{1-\left(\frac{x}{a}\right)^{2}}\right)^{(2-\lambda) / 2},
\end{aligned}
$$

with an eigenvalue

$$
E_{0}=\mu^{2}\left(8\left(3 g_{1}-2 g_{2}\right)-(\lambda-1)^{2}\right)
$$

Nonetheless, the boundary condition on the wave function

$$
\psi(x)=m(x)^{1 / 4} \phi(q(x))
$$

on the $x$-axis would mandate

$$
\lim _{x \rightarrow \pm a} \psi(x)=0
$$

Feasibly, the satisfaction of this condition is obviously achievable when $\lambda>2$. This would imply that

$$
\lambda=\frac{1}{2}\left(1+\sqrt{1+80 g_{1}-64 g_{2}}\right)>2
$$

At this point, we choose to stick with the continuity conditions at the heterojunction boundaries between two crystals and take $\alpha=\gamma \Rightarrow \beta=-1-2 \alpha$ to imply

$$
\lambda=\frac{1}{2}\left(1+\sqrt{(3+8 \alpha)^{2}}\right)>2 \Longrightarrow|3+8 \alpha|>3 .
$$

This suggests, in addition to von Roos constraint (2), new constraints $\alpha=$ $\gamma<-3 / 4$ or $\alpha=\gamma>0$ on the ambiguity parameters. In table 1 , we list the 
parametric values associated with the available orderings in the literature, along with their classifications as to being admissible (i.e., $\lambda>2$ ) or non-admissible (i.e., $\lambda \leq 2$ ). In the same table, we list some new orderings that are feasibly admissible. Obviously, non of the orderings known in the literature can be labeled as admissible, i.e., $\lambda>2$, (within our current methodical proposal, of course).

\section{Concluding Remarks}

We have considered the PDM-von Roos Hamiltonian for a quantum particle endowed with an unusual position-dependent mass function with two singularities in an infinite potential well. A point-canonical-transformation is used and an obvious correspondence between two effective (reference and target) Hamiltonians is obtained. Apparently, the singular PDM settings led to (through a PCT) the reflectionless modified Pöschl-Teller potential well. The exact solution of which is known in different perspectives. We have followed Diaz et al. [25] to come out with the result that non of the available known orderings in the literature is labeled admissible. Hereby, it should be noted that, our classifications on the "admissibility" and "non-admissibility" of the ordering ambiguity parameters (reported in table 1) are consequences of the boundary condition in (23). The validity of which resides only within the setting of the current methodical proposal associated with our model in (4).

Nevertheless, one could re-scale $k^{2}$ of equation (2) of Lekner [21] for $\lambda=\nu+1$, for example, and deduce all related reflectionless positive energy states, nonreflecting wave packets, etc. Yet, by re-scaling $k^{2}$ of equation (39.2) of Flügge [37] for $\lambda=\nu+1$, one would recycle Flügge's results. Consequently, we may conclude that the ordering ambiguity conflict associated with the uniqueness of the kinetic energy operator does not only depend on the heterojunction boundaries 
between two crystals and the Dutra's and Almeida's [14] reliability test. Even the form of the potential and/or the form of the position dependent mass may have their say in the process, so to speak. Such ambiguity in the non-uniqueness representation of the position-dependent mass Schrödinger Hamiltonian should be attributed to the lack of the Galilean invariance (cf., e.g., ref.[33] for more details on the issue).

To the best of our knowledge, such a study has not been considered elsewhere, not only within the Hermitian PDM-Hamiltonians' settings, but neither within the complex non-Hermitian settings. A gap that remains interesting and merits exploration/attention. 


\section{References}

[1] A Puente, M Casas, Comput. Mater Sci. 2 (1994) 441

[2] G Bastard, Wave Mechanics Applied to Semiconductor Heterostructures, Les Editions de Physique, Les Ulis, 1988.

[3] L I Serra, E Lipparini, Europhys. Lett. 40 (1997) 667.

[4] F Arias de Saaverda, J Boronat, A Polls, A Fabrocini, Phys. Rev. B 50 (1994) 4248 .

[5] M Barranco, M Pi, S M Gatica, E S Hemandez, J Navarro, Phys. Rev. B 56 (1997) 8997.

[6] A Puente, L I Serra, M Casas, Z. Phys. D 31 (1994) 283.

[7] A R Plastino, M Casas, A Plastino, Phys. Lett. A281 (2001) 297.

[8] A Schmidt, Phys. Lett. A 353 (2006) 459.

[9] S H Dong, M Lozada-Cassou, Phys. Lett. A 337 (2005) 313.

[10] I O Vakarchuk, J. Phys. A; Math. Gen. 38 (2005) 4727.

[11] C Y Cai, Z Z Ren, G X Ju, Commun. Theor. Phys. 43 (2005) 1019.

[12] B Roy, P Roy, Phys. Lett. A 340 (2005) 70.

[13] B Gonul, M Kocak, Chin. Phys. Lett. 20 (2005) 2742.

[14] A de Souza Dutra, C A S Almeida, Phys Lett. A 275 (2000) 25.

[15] T Gora, F Williams, Phys. Rev. 177 (1969) 1179.

[16] D J Ben Danial, and C B Duke, Phys. Rev. 152 (1966) 683.

[17] Q G Zhu, H Kroemer, Phys. Rev. B 27 (1983) 3519. 
[18] T Li, K J Kuhn, Phys. Rev. B 47 (1993) 12760.

[19] O Mustafa, S.Habib Mazharimousavi, Int. J. Theor. Phys 46 (2007) 1786.

[20] S. Cruz y Cruz, J Negro, L. M. Nieto, Phys. Lett. A 369 (2007) 400.

[21] J Lekner, Am. J. Phys. 75 (2007) 1151

[22] C Quesne, V M Tkachuk, J. Phys. A: Math. Gen. 37 (2004) 4267.

[23] L Jiang, L Z Yi, C S Jia, Phys. Lett. A 345 (2005) 279.

[24] O Mustafa, S H Mazharimousavi, Phys. Lett. A 358 (2006) 259.

[25] J I Diaz, J Negro, L M Nieto, O Rosas-Ortiz, J Phys A; Math. Gen. 32 (1999) 8447

[26] A D Alhaidari, Phys. Rev. A 66 (2002) 042116.

[27] O Mustafa, S H Mazharimousavi, J. Phys. A: Math. Gen. 39 (2006) 10537.

[28] B Bagchi, A Banerjee, C Quesne, V M Tkachuk, J. Phys. A; Math. Gen. 38 (2005) 2929.

[29] J Yu, S H Dong, Phys. Lett. A 325 (2004) 194.

[30] C Quesne, Ann. Phys. 321 (2006) 1221.

[31] T Tanaka, J. Phys. A; Math. Gen. 39 (2006) 219.

[32] Dutra A de Souza, J. Phys. A; Math. Gen. 39 (2006) 203.

[33] O von Roos, Phys. Rev. B 27 (1983) 7547.

[34] O Mustafa, S H Mazharimousavi, Czech. J. Phys 56 (2006) 297

[35] O Mustafa, S H Mazharimousavi, Phys. Lett. A 357 (2006) 295

[36] O Mustafa, S H Mazharimousavi, J Phys A: Math. Theor.41 (2008) 244020 
[37] S. Flügge, Practical Quantum Mechanics, Springer-Verlage, Berline, 1974; Problem 39 
Table 1: The parametric values associated with the available orderings in the literature, along with their classifications as to being admissible (i.e., $\lambda>2$ ) or non-admissible (i.e., $\lambda \leq 2$ ). New admissible values are also suggested.

\begin{tabular}{|c|c|c|c|c|c|}
\hline Available ordering & $\alpha=\gamma$ & $\beta$ & $\lambda$ & $E$ & Admissibility \\
\hline $\begin{array}{c}\text { Zhu and Kroemer [17], } \\
\text { and Li and Kuhn [18] }\end{array}$ & $-\frac{1}{2}$ & 0 & 1 & $\frac{1}{4}$ & non-admissible \\
\hline Gora and Williams [15] & - & 0 & - & - & non-admissible \\
\hline Ben Danial and Duke [16] & 0 & -1 & 2 & 0 & non-admissible \\
\hline Mustafa and Mazharimousavi [19] & $-\frac{1}{4}$ & $-\frac{1}{2}$ & 1 & 0 & non-admissible \\
\hline New & -1 & 1 & 3 & 5 & admissible \\
\hline New & $\frac{1}{4}$ & $-\frac{3}{2}$ & 3 & 0 & admissible \\
\hline New & $\frac{1}{2}$ & -2 & 4 & 0 & admissible \\
\hline New & $\frac{3}{4}$ & $-\frac{5}{2}$ & 5 & 0 & admissible \\
\hline New & 1 & -2 & 6 & 0 & admissible \\
\hline
\end{tabular}

